\title{
Electromagnetic couplings of radially excited light vector mesons from QCD sum rules
}

\author{
S. S. Afonin and T. D. Solomko \\ Saint Petersburg State University, 7/9 Universitetskaya nab., St.Petersburg, \\ 199034, Russia
}

\begin{abstract}
The couplings of unflavored vector mesons to the $e^{+} e^{-}$annihilation are an important source of information on the nature and structure of these resonances which play a prominent role in the hadron phenomenology. The couplings of many radially excited heavy vector mesons are measured, while the corresponding couplings for light vector mesons are not known. We propose a phenomenological method allowing to estimate these unknown couplings. The method is suggested by our observation that the electromagnetic coupling of the $n$-th radial excitation of $S$-wave heavy vector meson decouples from the $e^{+} e^{-}$annihilation with nearly exponential rate with $n$. It becomes natural to assume that the same effect takes place in the light vector mesons and this would allow to estimate the unknown couplings. We tested this assumption with the help of a generalized version of borelized QCD spectral sum rules saturated by a linear radial trajectory of meson states taken from the phenomenology. This leads to a consistent setup which is able to predict the decoupling rate. The calculated rate turned out to be almost the same as in the heavy vector mesons. Our result may be interpreted as an effective account for non-trivial electromagnetic formfactor in meson decays to $e^{+} e^{-}$, thus looking beyond the large- $N_{c}$ limit. On the one hand, we argue that the given decoupling does not necessary contradict to the previous large- $N_{c}$ results for the Regge like meson spectra.
\end{abstract}

\section{Introduction}

The Neutral Vector Mesons (NVMs) play a very important role in the hadron physics due to their direct coupling to photons. As is well known the highenergy photons interact with hadrons mainly by means of conversion into a NVM while the contribution of straight interaction with hadron electric charge is almost negligible. This gave rise to the famous hypothesis of vector meson dominance [1 4]. In contrast to gapless photons the NVMs possess 
a discrete spectrum of radial excitations. The couplings to the $e^{+} e^{-}$annihilation of radially excited heavy NVMs are known from the measured electromagnetic decay widths [5]. The same couplings for the light NVMs, however, are not known except the ground $\rho^{0}, \omega$, and $\phi$ states. This uncertainty may cause serious problems for checking various theoretical models, especially for models formulated in the large- $N_{c}$ limit of QCD [6,7] where all meson resonances represent narrow and hence well defined states.

We should briefly explain the point. Some time ago it was quite popular to address the spectroscopy of light mesons via the large- $N_{c}$ extensions of QCD spectral sum rules (see, e.g., Refs. [8 36]). Let us consider the $\rho$-meson as an example. The basic theoretical object in this approach is the two-point correlator $\Pi\left(Q^{2}\right)$ defined by

$$
\Pi_{\mu \nu}=\left(q_{\mu} q_{\nu}-g_{\mu \nu} q^{2}\right) \Pi\left(Q^{2}\right),
$$

where $\Pi_{\mu \nu}$ represents the T-product of two vector currents interpolating the neutral $\rho^{0}$-meson,

$$
\begin{gathered}
\Pi_{\mu \nu}=i \int d^{4} x e^{i q x}\left\langle 0\left|\mathrm{~T}\left\{j_{\mu}(x), j_{\nu}(0)\right\}\right| 0\right\rangle, \\
j_{\mu}=\frac{1}{2}\left(\bar{u} \gamma_{\mu} u-\bar{d} \gamma_{\mu} d\right) .
\end{gathered}
$$

Here $u$ and $d$ are quark fields, and $q$ is the photon space-like momentum, $q^{2}=-Q^{2}$. In the large- $N_{c}$ limit, the correlator is saturated by one-hadron states,

$$
\Pi\left(q^{2}\right)=\sum_{n} \frac{F_{n}^{2}}{q^{2}-m_{n}^{2}+i \epsilon} .
$$

The resonances are regarded as infinitely narrow because the large- $N_{c}$ scaling of meson masses is $m_{n} \sim N_{c}^{0}$ while the scaling of full meson width is $\Gamma_{n} \sim$ $m_{n} / N_{c}$, so $\Gamma_{n} \rightarrow 0$ in the limit $N_{c} \rightarrow \infty$. The quantities $F_{n}$ in residues of relation (3) represent electromagnetic couplings defined by

$$
\left\langle\operatorname{vac}\left|j^{\mu}\right| \rho_{n}\right\rangle=\frac{1}{2} e F_{n} m_{n} \varepsilon_{n}^{\mu}
$$

where $\varepsilon_{n}^{\mu}$ is the polarization vector of the vector meson $\rho_{n}$. At large $q^{2}$ the leading perturbative contribution to $\Pi\left(q^{2}\right)$ is logarithmic,

$$
\Pi\left(q^{2}\right) \sim \log q^{2}
$$

It is clear that the consistency of Eq. (3) with Eq. (5) can be achieved only if infinite number of states are included in Eq. (3) [7]. 
For further analysis one needs to postulate the form of meson spectrum. Basing on various theoretical (starting from the Veneziano model, for more recent suggestions see, e.g., Refs. [37 52]) and phenomenological (see, e.g., Refs. [53 65] ) expectations the spectrum was usually interpolated by a simple linear Regge-like trajectory of radial states,

$$
m_{n}^{2}=a n+m_{0}^{2}, \quad n=0,1,2, \ldots,
$$

sometimes with some non-linear corrections.

In the case of linear ansatz (6), the logarithmic asymptotic (5) can be obtained only if $F_{n}^{2}$ in Eq. (3) behave with $n$ as

$$
F_{n}^{2} \sim \frac{d m_{n}^{2}}{d n} \sim \text { const }
$$

at least for $n \rightarrow \infty$. In this way one arrives at the standard large- $N_{c}$ prediction for the e/m constants $F_{n}$ : They are expected to be almost $n$-independent for large enough $n$, i.e. all highly excited states should couple to the $e^{+} e^{-}$ annihilation nearly equally. The problem is that we still cannot check this prediction - the e/m constants $F_{n}$ are related to the e/m decay widths,

$$
\Gamma_{\rho_{n} \rightarrow e^{+} e^{-}}=\frac{4 \pi \alpha^{2} F_{n}^{2}}{3 m_{n}}
$$

which have not been reliably measured for the radially excited states, $n>0$, in the light quark sector. A pertinent question of validity of relation (7) emerges also in the soft-wall holographic model of QCD [66] and in numerous extensions of this model (see, e.g., discussions in Refs. [67-71]). The given bottom-up holographic approach is congenial with the method of large- $N_{c}$ QCD sum rules [72,73] and the both are based on at least asymptotic validity of Eq. (7). In the situation of absence of a direct experimental information on important couplings $F_{n}$, may we test the prediction (7) somehow indirectly?

In the present work, we will give arguments disfavoring the validity of Eq. (7) in the real world with $N_{c}=3$ if the relation (86) holds.

The paper is organized as follows. In the introductory Section 1, we have formulated the problem. In Section 2, we recall briefly how the relation (7) was originally proposed and revise the original estimates. A comparison with the situation in heavy vector mesons is made in Section 3. Motivated by this comparison we propose in Section 4 an ansatz for $F_{n}^{2}$ and perform a test for this ansatz in the framework of QCD spectral sum rules. Concluding discussions on possible interpretations of our result are given in the final Section 5 . 


\section{Extended Vector Meson Dominance}

We find instructive to begin with a brief reminder of an old history of appearing the relation (17) from the hypothesis of extended Vector Meson Dominance (VMD). The classical VMD hypothesis put forward by Sakurai 74 ] states that the vector current in (2) can be replaced by the field of vector meson,

$$
j^{\mu}=e \frac{m_{\rho}^{2}}{2 f_{\rho}} \rho^{\mu} .
$$

The full vector current includes of course the field of $\omega$ and $\phi$ meson but this will be not essential for our purposes. The dimensionless coupling $f_{\rho}$ in (9) is related to $F_{\rho}$ in (44) as

$$
f_{\rho}=\frac{m_{\rho}}{F_{\rho}}
$$

We prefer to use temporary this traditional for the VMD hypothesis notation.

At the beginning of 1970s it was realized that the VMD works well at low enough energies and at higher energies the predictions can be improved if the second $\rho$-meson, the excited $\rho^{\prime}$ one, is added to (9). This resonance was referred to as $\rho(1600)$ [75. It was looking natural to include into the identity (9) further higher mass vector mesons up to infinite number. In particular, the authors of Ref. [76] (and independently Sakurai [77]) argued that the infinite number of states in (9) is able to describe the scaling of the inelastic structure functions. This alternative scheme to parton models has subsequently been interpreted as a particular manifestation of the quarkhadron duality. In this model, the total $e^{+} e^{-}$annihilation cross section into hadrons is given by

$$
\sigma_{e \bar{e} \rightarrow \mathrm{h}}(s)=\frac{4 \pi^{2} \alpha^{2}}{s^{\frac{3}{2}}} \sum_{n} \frac{m_{n}^{3}}{f_{n}^{2}} \frac{m_{n} \Gamma_{n}}{\left(s-m_{n}^{2}\right)^{2}+m_{n}^{2} \Gamma_{n}^{2}} .
$$

If asymptotically $\sigma_{e \bar{e} \rightarrow \mathrm{h}}(s)$ scales as $1 / s$ then the sum in (10) must behave as a function of c.m. energy $s$ as $\sqrt{s} \times$ const $\times \Theta\left(s-m_{0}^{2}\right)$ implying for large $n$

$$
\frac{m_{n}^{2}}{f_{n}^{2}} \rightarrow \text { const }
$$

which is the relation (77). The analysis of existed data in terms of a broad $\rho(1600)$ which was performed in Ref. [75] resulted in the estimate $f_{\rho^{\prime}} / f_{\rho} \approx \sqrt{5}$ (see below) suggesting that the relation (11) is approximately satisfied already for $\rho(1600)$. Indeed, we have from these numbers $\left(m_{\rho} / f_{\rho}\right)^{2} /\left(m_{\rho^{\prime}} / f_{\rho^{\prime}}\right)^{2} \approx$ 1.2. From this observation the authors of Ref. [76] assumed for simplicity that (11) is true for all $n$. 
Let us now revise the analysis of Ref. [75] using the modern data. The partial width of the $\rho^{\prime} \rightarrow \omega \pi \rightarrow 4 \pi$ decay mode was predicted to be

$$
\Gamma_{\rho^{\prime} \rightarrow \omega \pi}=2.4\left(\frac{f_{\rho^{\prime}}}{f_{\rho}}\right)^{2} \Gamma_{\omega \rightarrow 3 \pi}
$$

Making use of $S U(3)$ and phase-space evaluation techniques and substituting $\Gamma_{\omega \rightarrow 3 \pi} \approx 10 \mathrm{MeV}$ the authors of Ref. [75] obtained for the main $\rho^{\prime} \rightarrow \mathrm{VP}$ decay modes (in $\mathrm{MeV}$ )

$$
\Gamma_{\rho^{\prime} \rightarrow \omega \pi}=24\left(\frac{f_{\rho^{\prime}}}{f_{\rho}}\right)^{2}, \quad \Gamma_{\rho^{\prime} \rightarrow \rho \eta}=7\left(\frac{f_{\rho^{\prime}}}{f_{\rho}}\right)^{2}, \quad \Gamma_{\rho^{\prime} \rightarrow K^{*} K}=4\left(\frac{f_{\rho^{\prime}}}{f_{\rho}}\right)^{2} .
$$

The pseudoscalar-pseudoscalar decay modes were estimated to be equal to $\Gamma_{\rho^{\prime} \rightarrow \mathrm{PP}} \simeq 8\left(\frac{f_{\rho^{\prime}}}{f_{\rho}}\right)^{2} \mathrm{MeV}$. By adding all these rates they obtained $\Gamma_{\rho^{\prime} \rightarrow \mathrm{VP}, \mathrm{PP}} \simeq$ $43\left(\frac{f_{\rho^{\prime}}}{f_{\rho}}\right)^{2} \mathrm{MeV}$. Using $\Gamma_{\rho^{\prime}} \simeq 215 \mathrm{MeV}$ the final result was $\left(f_{\rho^{\prime}} / f_{\rho}\right)^{2} \approx 5$.

The modern value for the width of decay $\omega \rightarrow 3 \pi$ is $\Gamma_{\omega \rightarrow 3 \pi} \approx 7.57 \mathrm{MeV}[5]$. Thus the numbers in (13) should be multiplied by the factor of 0.757 and this leads to $\Gamma_{\rho^{\prime}} \simeq 34\left(\frac{f_{\rho^{\prime}}}{f_{\rho}}\right)^{2} \mathrm{MeV}$.

Since 1988 the old resonance $\rho(1600)$ has been replaced in PDG by two resonances $\rho(1450)$ and $\rho(1700)$ [5]. The former is usually interpreted as the first radial $S$-wave excitation of $\rho(770)$. The substitution of $\Gamma_{\rho(1450)}=400 \pm$ $60 \mathrm{MeV}$ and $m_{\rho(1450)}=1465 \pm 25 \mathrm{MeV}[5]$ leads to the estimate $\left(f_{\rho^{\prime}} / f_{\rho}\right)^{2} \approx$ $11.6 \pm 1.7$. With the modern data we hence obtain $\left(m_{\rho} / f_{\rho}\right)^{2} /\left(m_{\rho^{\prime}} / f_{\rho^{\prime}}\right)^{2} \approx$ $3.2(3)$ instead of 1.2 above. Thus the relation (11) is in a sharp contradiction for $\rho^{\prime}$. Rephrasing this in terms of couplings $F_{n}$ in (7) we have the estimate,

$$
\beta \equiv \frac{F_{1}^{2}}{F_{0}^{2}} \approx 0.31(3)
$$

It is interesting to compare the given value of $\beta$ with other estimates existing in the literature. We found some estimates for $F_{1}$ (i.e. for $F_{\rho^{\prime}}$ ) which lead to the following predictions: $\beta \approx 0.3$ [78] (from a model of $S$-matrix unitarity for overlapping resonances); $\beta \gtrsim 0.3$ [79] (from contribution of $\rho^{\prime}$ to the electromagnetic pion mass difference); $\beta \approx 0.25$ [80] (from a specific model of gluon vacuum); $\beta \approx 0.27-0.46$ [81] (from a specific relativized potential quark model); $\beta \approx 0.59(12)$ [82] (from finite energy QCD sum rules saturated by two resonances). It should be mentioned that the last estimate obtained with the help of the least square fitting method is consistent with the estimates which we will get in the present paper. 


\section{A hint from the heavy quark sector}

The subject discussed in the previous Section is almost 50 years old. However we still do not have any significant progress in getting reliable experimental data on e/m couplings of light excited NVMs. Such a situation looks unfortunate to say the least. On the other hand, a serious progress was achieved in the heavy NVMs. The vector charmonia and bottomonia represent direct analogues of light $\rho^{0}, \omega$, and $\phi$ mesons and share common properties. It is worth to mention a recent observation that even their radial Regge trajectories look very similar if one subtracts the quark masses $m_{q}$ from the corresponding masses of heavy mesons: $\left(m_{n}-2 m_{q}\right)^{2}=a n+m_{0}^{2}$, where the slope $a$ and intercept $m_{0}^{2}$ are nearly universal for both light and heavy mesons 83 86. It looks natural to expect that their electromagnetic properties are also similar.

The $e^{+} e^{-}$decay width of many excited $\psi$ and $\Upsilon$ mesons was measured with a satisfactory accuracy [5]. By extracting the e/m couplings $F_{n}$ from these data with the help of relation (8) we observe that the prediction (7) is not fulfilled. One has instead a nearly exponential decrease with $n$. To quantify this observation we will interpolate the given decrease in the form of geometrical progression,

$$
\beta^{n} \equiv \frac{F_{n}^{2}}{F_{0}^{2}}=\frac{\Gamma_{e \bar{e}, n} m_{n}}{\Gamma_{e \bar{e}, 0} m_{0}},
$$

where $n=0$ refers to the ground state. In Table 1 we show the extracted values of $\beta$ for those vector mesons which are supposed to be the $S$-wave radial excitations of $J / \psi(1 S)$ and $\Upsilon(1 S)$ states.

Table 1: The masses, $e^{+} e^{-}$decay widths and values of $\beta$ for the $S$-wave heavy vector mesons.

\begin{tabular}{|c||c|c|c|c|c|}
\hline & $J / \psi(1 S)$ & $\psi(2 S)$ & $\psi(4040)$ & $\psi(4160)$ & $\psi(4415)$ \\
\hline \hline $\mathrm{m}, \mathrm{MeV}$ & 3097 & 3686 & $4039 \pm 1$ & $4191 \pm 5$ & $4421 \pm 4$ \\
\hline$\Gamma_{e \bar{e}}, \mathrm{keV}$ & $5.55 \pm 0.14$ & $2.34 \pm 0.04$ & $0.86 \pm 0.07$ & $0.48 \pm 0.22$ & $0.58 \pm 0.07$ \\
\hline$\beta$ & - & $0.50 \pm 0.02$ & $0.45 \pm 0.03$ & $0.49 \pm 0.08$ & $0.62 \pm 0.02$ \\
\hline
\end{tabular}

\begin{tabular}{|c||c|c|c|c|c|}
\hline & $\Upsilon(1 S)$ & $\Upsilon(2 S)$ & $\Upsilon(3 S)$ & $\Upsilon(4 S)$ & $\Upsilon(11020)$ \\
\hline \hline $\mathrm{m}, \mathrm{MeV}$ & 9460 & 10023 & 10355 & $10579 \pm 1$ & $10988_{-3}^{+11}$ \\
\hline$\Gamma_{e \bar{e}}, \mathrm{keV}$ & $1.34 \pm 0.02$ & $0.61 \pm 0.01$ & $0.44 \pm 0.01$ & $0.27 \pm 0.03$ & $0.13 \pm 0.03$ \\
\hline$\beta$ & - & $0.48 \pm 0.01$ & $0.60 \pm 0.01$ & $0.61 \pm 0.02$ & $0.58 \pm 0.04$ \\
\hline
\end{tabular}


It is well seen that the values of $\beta$ are rather stable and concentrate near $\beta \approx 0.5-0.6$. This property is at odds with the large- $N_{c}$ prediction $\beta \approx 1$ in (17). It is tempting to assume that in the light quark sector the situation can be similar. The given assumption, in principle, can be tested within the framework of QCD spectral sum rules. In the next Section, we propose such a test.

\section{Electromagnetic couplings from QCD sum rules}

Let us substitute the ansatz (14) into the classical SVZ sum rules [87] extended to the case of arbitrary number of resonances (often called "the finite energy sum rules"). The method of QCD sum rules is based on the Operator Product Expansion (OPE) of the two-point correlator (2) in Euclidean space,

$$
\begin{aligned}
\Pi\left(Q^{2}\right)=\frac{1}{8 \pi^{2}}\left[\left(1+\frac{\alpha_{s}}{\pi}\right) \ln \frac{\mu^{2}}{Q^{2}}-\frac{6 m_{q}^{2}}{Q^{2}}\right] \\
+\frac{\left\langle m_{q} \bar{q} q\right\rangle}{Q^{4}}+\frac{1}{24 Q^{4}}\left\langle\frac{\alpha_{s}}{\pi}\left(G_{\mu \nu}^{a}\right)^{2}\right\rangle-\frac{14}{9} \frac{\pi \alpha_{s}}{Q^{6}}\langle\bar{q} q\rangle^{2},
\end{aligned}
$$

where $q$ stands for $u$ or $d$ quark, the coefficient in front of the last term is given in the large- $N_{c}$ limit, and further $\mathcal{O}\left(Q^{-8}\right)$ terms are neglected. The non-perturbative power corrections in the second line are given in form of various vacuum condensates. To improve the convergence of the OPE and increase the relative contribution of the ground state one applies the Borel transform,

$$
L_{M} \Pi\left(Q^{2}\right)=\lim _{\substack{Q^{2}, n \rightarrow \infty \\ Q^{2} / n=M^{2}}} \frac{1}{(n-1) !}\left(Q^{2}\right)^{n}\left(-\frac{d}{d Q^{2}}\right)^{n} \Pi\left(Q^{2}\right),
$$

to the OPE (15) and gets

$$
\begin{aligned}
L_{M} \Pi\left(Q^{2}\right)=\frac{1}{8 \pi^{2}}(1+ & \left.\frac{\alpha_{s}}{\pi}-\frac{6 m_{q}^{2}}{M^{2}}\right) \\
& +\frac{\left\langle m_{q} \bar{q} q\right\rangle}{M^{4}}+\frac{1}{24 M^{4}}\left\langle\frac{\alpha_{s}}{\pi}\left(G_{\mu \nu}^{a}\right)^{2}\right\rangle-\frac{7}{9} \frac{\pi \alpha_{s}}{M^{6}}\langle\bar{q} q\rangle^{2} .
\end{aligned}
$$

The vector correlator $\Pi\left(Q^{2}\right)$ satisfies a dispersion relation with one subtraction,

$$
\Pi\left(q^{2}\right)=\frac{1}{\pi} \int_{4 m_{q}^{2}}^{\infty} d s \frac{\operatorname{Im} \Pi(s)}{s-q^{2}+i \varepsilon}+\Pi(0) .
$$


In the classical SVZ sum rules, the correlator (18) is saturated by one resonance plus perturbative continuum. We will saturate by arbitrary number of states with the linear spectrum (6). The decay width in this method is neglected (aside from the large- $N_{c}$ arguments, this approximation can be motivated phenomenologically by the observation that on average the full width scales as $\Gamma_{n} \simeq 0.1 m_{n}[64,65$, so one expects an accuracy on the level of $10 \%$ ). We assume that the contribution of higher mass states is rapidly decreasing. This allows not to bother about an exact energy cutoff and use instead an infinite number of states. We will see the consistency of this assumption aposteriori.

In the case of ansatz (14), the imaginary part of $\Pi\left(Q^{2}\right)$ in the resonance representation (3) takes the form

$$
\operatorname{Im} \Pi\left(q^{2}\right)=\sum_{n} \pi \beta^{n} F_{0}^{2} \delta\left(q^{2}-m_{n}^{2}\right)
$$

The Borel transform of (18) is [87]:

$$
L_{M} \Pi\left(Q^{2}\right)=\frac{1}{\pi M^{2}} \int_{0}^{\infty} e^{-s / M^{2}} \operatorname{Im} \Pi(s) d s=\frac{F_{0}^{2}}{M^{2}} \sum_{n} \beta^{n} e^{-m_{n}^{2} / M^{2}},
$$

where we neglected the $\mathcal{O}\left(m_{q}^{2}\right)$ contribution. Substituting the linear spectrum (6) and summing the contributions from infinite number of states we obtain

$$
L_{M} \Pi\left(Q^{2}\right)=\frac{F_{0}^{2}}{M^{2}} \frac{e^{-m_{0}^{2} / M^{2}}}{1-\beta e^{-a / M^{2}}} .
$$

The first sum rule follows from equating the relations (15) and (21),

$$
\begin{aligned}
\frac{F_{0}^{2} e^{-m_{0}^{2} / M^{2}}}{1-\beta e^{-a / M^{2}}}= & \frac{M^{2}}{8 \pi^{2}}\left[1+\frac{\alpha_{s}}{\pi}-\frac{6 m_{q}^{2}}{M^{2}}\right. \\
& \left.+\frac{8 \pi^{2}}{M^{4}}\left\langle m_{q} \bar{q} q\right\rangle+\frac{\pi^{2}}{3 M^{4}}\left\langle\frac{\alpha_{s}}{\pi}\left(G_{\mu \nu}^{a}\right)^{2}\right\rangle-\frac{56}{9} \frac{\pi^{3} \alpha_{s}}{M^{6}}\langle\bar{q} q\rangle^{2}\right] .
\end{aligned}
$$

The second sum rule arises after taking derivative of Eq. (22) with respect to $1 / M^{2}$ [87]. The mass squared of the ground state appears directly in the fraction $-\frac{d(22)}{d\left(1 / M^{2}\right)} /(22)$,

$$
m_{0}^{2}=M^{2} \frac{h_{0}-\frac{h_{2}}{M^{4}}-\frac{2 h_{3}}{M^{6}}}{h_{0}+\frac{h_{1}}{M^{2}}+\frac{h_{2}}{M^{4}}+\frac{h_{3}}{M^{6}}}-\frac{a}{1-\beta e^{-a / M^{2}}},
$$

where the condensate terms $h_{i}$ are presented in Table 2 . 
Table 2: The condensate contributions $h_{i}$ and their numerical values used in our work (see text).

\begin{tabular}{|c|c|c|c|c|}
\hline & $h_{0}$ & $h_{1}$ & $h_{2}$ & $h_{3}$ \\
\hline Theor. & $1+\frac{\alpha_{s}}{\pi}$ & $-6 m_{q}^{2}$ & $8 \pi^{2}\left\langle m_{q} \bar{q} q\right\rangle+\frac{\pi^{2}}{3}\left\langle\frac{\alpha_{s}}{\pi}\left(G_{\mu \nu}^{a}\right)^{2}\right\rangle$ & $-\frac{56}{9} \pi^{3} \alpha_{s}\langle\bar{q} q\rangle^{2}$ \\
\hline Numer. & 1 & 0 & 0.032 & -0.030 \\
\hline
\end{tabular}

The first term in the r.h.s. of Eq. (23) corresponds to the limit $s_{0} \rightarrow \infty$ in the canonical expressions for the meson masses in the SVZ sum rules [87]. The energy cutoff $s_{0}$ is infinite in our case as we take into account the infinite number of radial excitations. The second term reflects contribution of highly excited states $(a \neq 0)$ with, generally speaking, decreasing residues in the corresponding poles $(0<\beta \leq 1)$.

We will set $h_{0}=1$ since taking the perturbative threshold $s_{0} \rightarrow \infty$ (infinite number of radial states) we should have $\alpha_{s} \rightarrow 0$ due to the asymptotic freedom. This is consistent with the Borel transform (16). Indeed, the coupling $\alpha_{s}$ is a function of the momentum injected - the corresponding one-loop expression in Euclidean space is $\alpha_{s} \sim 1 / \ln \frac{Q^{2}}{\Lambda_{\mathrm{QCD}}^{2}}$. In finite energy sum rules, one neglects this slow running of $\alpha_{s}$ with $Q^{2}$ setting $\mu^{2}=s_{0}$. But in the infinite energy region this is not a good approximation and the running of $\alpha_{s}$ must be taken into account. The induced correction to the unit operator in OPE will be then proportional to $\ln \frac{\mu^{2}}{Q^{2}} / \ln \frac{Q^{2}}{\Lambda_{\mathrm{QCD}}^{2}}$. One can see that this term becomes zero after applying the Borel transform (16) its leading part is proportional to $\lim _{Q^{2} \rightarrow \infty} \ln \frac{\mu^{2}}{\Lambda_{\mathrm{QCD}}^{2}} / \ln ^{2} \frac{Q^{2}}{\Lambda_{\mathrm{QCD}}^{2}}$. For the same reason other loop corrections to the unit operator in the OPE disappear after the Borel transform.

Now we should fix the other input parameters. The values of gluon and quark condensates are taken from the work [87]: $\left\langle\frac{\alpha_{s}}{\pi}\left(G_{\mu \nu}^{a}\right)^{2}\right\rangle=(330 \mathrm{MeV})^{4}$, and $\langle\bar{q} q\rangle=-(250 \mathrm{MeV})^{3}$. The first value is scale-independent while the second one is taken roughly at the scale $\mu=1 \mathrm{GeV}$. From the Gell-MannOakes -Renner relation, $m_{\pi}^{2} f_{\pi}^{2}=-\left(m_{u}+m_{d}\right)\langle\bar{q} q\rangle$, with the pion mass $m_{\pi}=140 \mathrm{MeV}$ [5] and pion weak decay constant $f_{\pi}=92.4 \mathrm{MeV}$, one gets $m_{u}+\left.m_{d} \approx 10.7 \mathrm{MeV}\right|_{\mu=1 \mathrm{GeV}}$. We consider the isospin limit for the masses of current quarks, $m_{u}=m_{d} \equiv m_{q}$. Thus we get a numerical value for another renormalization invariant condensate of dimension four, $\left\langle m_{q} \bar{q} q\right\rangle=-(95.6 \mathrm{MeV})^{4}$. The scale-dependent term $\mathcal{O}\left(m_{q}^{2}\right)$ is numerically 
very small and should be neglected within our accuracy. All these inputs lead to the values of $h_{1}$ and $h_{2}$ in Table 2. The operator $\alpha_{s}(\bar{q} q)$ has a small anomalous dimension. We will regard the corresponding v.e.v. $\alpha_{s}\langle\bar{q} q\rangle$ as a constant. The numerical value for $h_{3}$ in Table 2 is taken from Ref. [87].

The last free parameter to be fixed is the slope $a$ in the linear spectrum (6). The fixation of slope from the data on excited $\rho$-mesons is somewhat ambiguous as these data admit various interpretations [5]. We will stick with a conservative point and take a value averaged over many linear trajectories [54, $a=1.14 \mathrm{GeV}^{2}$.

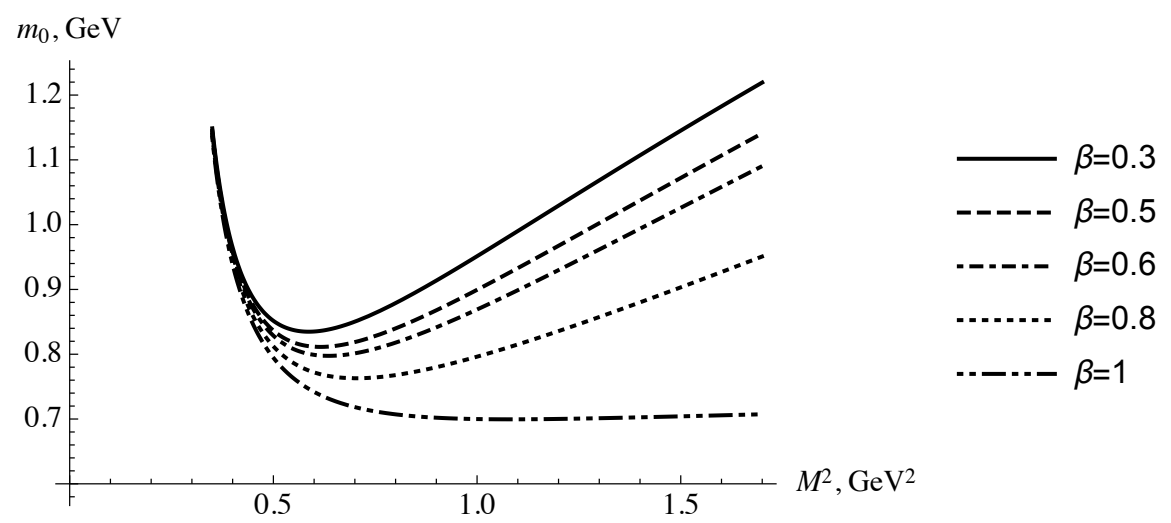

Figure 1: The mass of $\rho$ meson on the Borel plane at different values of $\beta$.

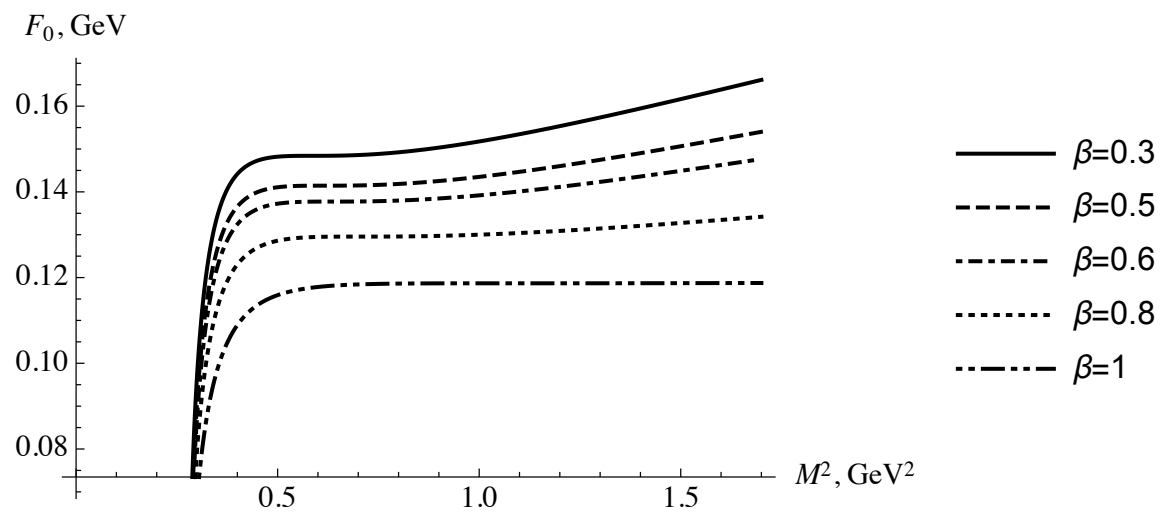

Figure 2: The e/m constant of $\rho$ meson on the Borel plane at different values of $\beta$.

The behavior of ground state mass $m_{0}$ calculated from Eq. (23) and of $\mathrm{e} / \mathrm{m}$ coupling $F_{0}$ from Eq. (22) as a function of Borel parameter $M^{2}$ at different values of $\beta$ is displayed on Fig. 1 and Fig. 2 respectively. The existence of stability region — the so-called "Borel window" — is well seen for 
$0.5<M^{2}<0.8 \mathrm{GeV}^{2}$ when $\beta<1$. The predictions of SVZ sum rule method refer to that stability region only. It should be remarked that we used the narrow-width approximation where the values of $m_{0}$ and $F_{0}$ may be different from the experimental ones, $m_{\rho}=775 \mathrm{MeV}$ and $F_{\rho}=156(2) \mathrm{MeV}$ [5]. For instance, the unitarized chiral perturbation theory predicts the enhancement of $m_{\rho}$ by $40-60 \mathrm{MeV}$ when taking the zero-width limit [88 90]. On the other hand, the Regge phenomenology of light and heavy vector mesons suggests that their ground states lie always below the corresponding radial linear trajectories [83 86, i.e. the linear ansatz (6) should predict a somewhat enhanced value for $m_{0}$. Since we made use of both the zero-width approximation and the linear radial trajectories, we should reproduce the value of $m_{0}$ expected in these approximations, i.e., roughly speaking, we should use $m_{\rho}=800-830 \mathrm{MeV}$ as a reference value. The given mass is achieved near $\beta \approx 0.5-0.6$ (see Fig. 1). Exactly this interval of $\beta$ was observed for the heavy vector mesons in the previous Section! This is our main result.

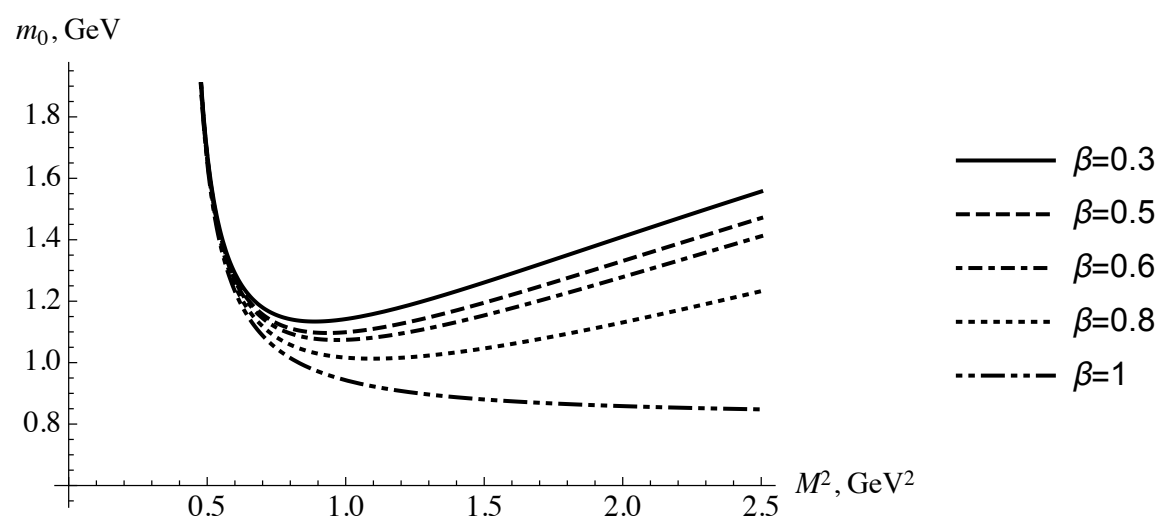

Figure 3: The mass of $\phi$ meson on the Borel plane at different values of $\beta$.

The same analysis can be carried out for the $\omega$ and $\phi$ mesons. The case of $\omega$ is numerically almost identical to the $\rho$ one. In the $\phi$ channel, we take $m_{s}=130 \mathrm{MeV}$ for the mass of strange quark at $1 \mathrm{GeV}[5$ ] and the same value of slope $a\left[83\right.$ 86]. After this substitution (and account for the $6 m_{s}^{2} / Q^{2}$ correction to the bare quark loop which was neglected in the $\rho$ channel), the values of $m_{\phi}\left(M^{2}\right)$ and $F_{\phi}\left(M^{2}\right)$ are shown on Fig. 3 and Fig. 4 respectively. It is seen that the predicted value of $\beta$ seems to be close to that predicted for $\rho$ mesons.

We should remark, however, that the issue of large- $N_{c}$ masses is not well settled and the phenomenology of linear radial trajectories can vary from paper to paper. For this reason we would provide also a more conservative estimate for the rate of decoupling: $\beta \approx 0.3-0.8$ as is shown in Figs. 1 and 3 . 


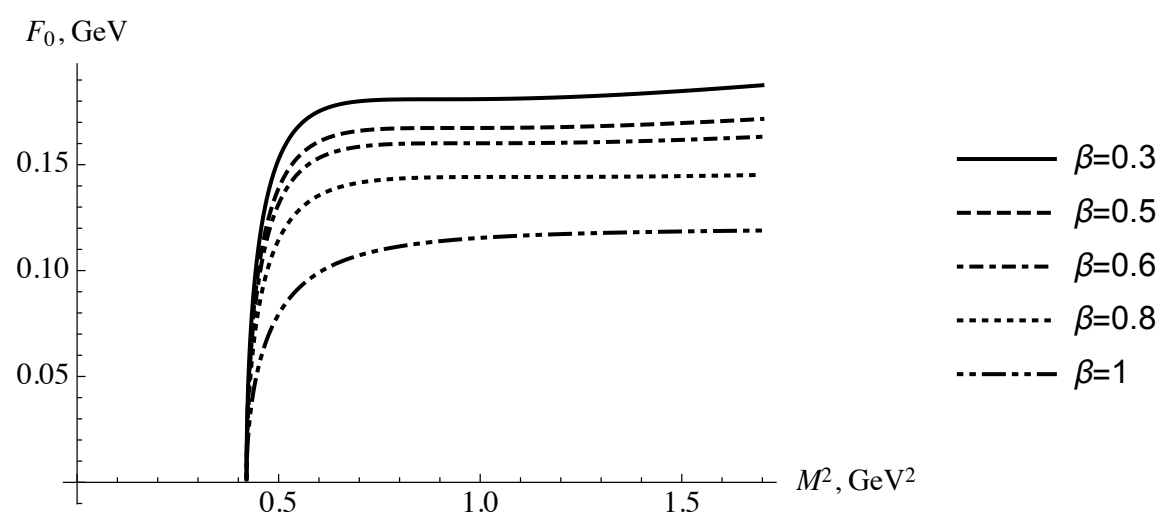

Figure 4: The constant $F_{\phi}$ on the Borel plane at different values of $\beta$.

We believe that this broader interval absorbs other theoretical uncertainties present in our analysis. First of all, we used strictly linear ansatz (6) for the mass spectrum. The large- $N_{c}$ QCD sum rules with non-linear corrections to the ansatz (66) were considered in many papers [10-18]. The experience of those models shows that the impact of non-linear corrections competes with the accuracy of the large- $N_{c}$ approximation itself. Such corrections can be added to our analysis as well but at the price of introducing new parameters. We think that this would not make our results substantially more convincing. Another issue is the influence of approximating the sum over hadron states in Eq. (3) by a infinite sequence of stable one-meson poles. What if we relax in a reasonable manner these tight bounds? One usually relates the caused uncertainties with violations of local quark-hadron duality. There is no theory of such violations, only models (see, e.g., Refs. [8,10 12]). The violations might become important at finite $N_{c}$. Within the proposed models, the residues usually acquire additional factor $F^{2} \rightarrow F^{2} /\left(1+K / N_{c}\right)$, with constant $K>0$. Since we dealt with fractions of residues, this extra factors are not relevant for our analysis. Also the spectral density acquires additional contribution, up to a normalization factor the spectral function looks like [8]

$$
\rho\left(q^{2}\right)=\frac{1}{\pi} \operatorname{Im} \Pi\left(q^{2}\right) \rightarrow 1+\text { power corr. }+2 e^{-A q^{2} / N_{c}} \cos \left(B q^{2}\right),
$$

where $A, B>0$ are some constants. The last term is present even in the limit of infinite $N_{c}$, but it oscillates and therefore is not seen in the OPE in Euclidean space. It becomes exponentially damped at finite $N_{c}$ so it should affect only slightly the physical observables in the Minkowski space at $N_{c}=3$. The quantitative impact depends on a concrete observable. For instance, the overall theoretical uncertainty caused by duality violations for 
the cross section of hadronic $\tau$-decays was estimated in Ref. [8] on the level of $3 \%$. We recall that the expected theoretical uncertainty from the large- $N_{c}$ approximation is on the level of $10-20 \%$. This should definitely absorb the uncertainties coming from duality violations.

\section{Concluding discussions}

We demonstrated that the exponential decrease of couplings of light vector mesons to the $e^{+} e^{-}$annihilation as a function of radial number $n$ is consistent with the QCD spectral sum rules and estimated numerically the rate of decrease. This rate turned out to be close (perhaps equal) to that in the heavy vector mesons. The physical meaning of our result is that the radially excited neutral vector mesons seem to decouple from the $e^{+} e^{-}$annihilation exponentially fast with $n$ and the mechanism of this decoupling is likely universal for the light and heavy mesons.

The highly excited radial states of light vector mesons are practically not seen (roughly starting from the second radial excitation) in the $e^{+} e^{-}$annihilation into hadrons and it became standard to attribute this to rapidly growing and overlapping decay widths as is modeled in the ansatz (10). The representation in form of a sum of Breit-Wigner peaks is known to contradict to analytical properties of amplitudes but we draw attention to another problem: Even "corrected" versions of such a representation (see Ref. [8]) predict a much slower decrease of the height of resonance peaks in the crosssection of $e^{+} e^{-}$annihilation to hadrons. Our calculation suggests that the dominant contribution to this "melting" of excited states in the perturbative continuum may arise from the proposed effect of exponential decoupling.

Our conclusion does not necessary contradict to the large- $N_{c}$ prediction (7). We considered a finite number of states in QCD sum rules, i.e. finite $N_{c}$, and the replacement by infinite number of states in the sum was just a technical approximation which was justified owing to a fast decrease of residues. One can easily imagine a situation when both pictures are mutually consistent. For instance, the residues $F_{n}^{2}$ may contain an $N_{c}$-dependent factor $\left(e^{-2 / N_{c}}\right)^{n}$ which is equal to 1 in the limit $N_{c} \rightarrow \infty$ (thus leading to the prediction (7) $)$ and to $\left(e^{-2 / 3}\right)^{n} \approx 0.5^{n}$ in the real world as we obtained. In this scenario, one cannot neglect the $1 / N_{c}$ corrections to e/m couplings of radially excited states since, in contrast to meson masses, this would lead to a dramatic disagreement with the hadron phenomenology.

The most plausible origin of decoupling under consideration is the well known fact that hadrons are very complicated extended objects. It means that hadron interaction with vector current must include an $\mathrm{e} / \mathrm{m}$ formfactor 
which may differ significantly in the large- $N_{c}$ limit and at finite $N_{c}=3$. On a qualitative level, the quarks inside excited states are much more energetic and, consequently, on average more separated in space. This should lead to decoupling from interaction with strictly local vector current since the couplings $F_{n}$ are related with the wave function of quark-antiquark pair at zero space separation, $F_{n} \sim \Psi_{n}(0)$. We have a freedom to ascribe the effect of non-trivial formfactor either to definition of e/m couplings $F_{n}$ or to definition of $e^{+} e^{-}$decay width (8) - its r.h.s. should be then multiplied by a factor $\mathcal{F}_{n}$ that reflects the impact of formfactor at different $n$,

$$
\Gamma_{\rho_{n} \rightarrow e^{+} e^{-}}=\frac{4 \pi \alpha^{2} F_{n}^{2}}{3 m_{n}} \mathcal{F}_{n}
$$

Within the former definition, we obtain decreasing with $n$ couplings $F_{n}$, as we did in the present work. Within the latter one, the couplings $F_{n}$ in (25)

are constant, $F_{n}=F_{0}$, in accord with the large- $N_{c}$ result (7), but predictions for $\Gamma_{\rho_{n} \rightarrow e^{+} e^{-}}$must be calculated with the factor $\mathcal{F}_{n}$ taken into account. We may reformulate the result of our analysis as a phenomenological derivation of this factor, $\mathcal{F}_{n} \approx e^{-\frac{2}{3} n}$.

The decoupling scheme proposed in the present work can have various applications. For instance, it would be interesting to employ it in the large$N_{c}$ calculations of electromagnetic pion (see, e.g., Ref. [9,91,92] and references therein) and proton [93] fomrfactors.

\section{References}

[1] J. Sakurai, Currents and mesons (University of Chicago Press, 1969).

[2] T. H. Bauer, R. D. Spital, D. R. Yennie, F. M. Pipkin, Rev. Mod. Phys. 50, 261 (1978) Erratum: [Rev. Mod. Phys. 51, 407 (1979)].

[3] H. B. O'Connell, B. C. Pearce, A. W. Thomas, A. G. Williams, Prog. Part. Nucl. Phys. 39, 201 (1997).

[4] S. Leupold, C. Terschlusen, PoS BORMIO 2012, 024 (2012) arXiv:1206.2253 [hep-ph]].

[5] C. Patrignani et al. (Particle Data Group), Chin. Phys. C 40, 100001 (2016).

[6] G. 't Hooft, Nucl. Phys. B 72, 461 (1974).

[7] E. Witten, Nucl. Phys. B 160, 57 (1979). 
[8] M. Shifman, arXiv:hep-ph/0009131.

[9] M. Shifman, arXiv:hep-ph/0507246.

[10] B. Blok, M. A. Shifman, D. X. Zhang, Phys. Rev. D 57, 2691 (1998) Erratum: [Phys. Rev. D 59, 019901 (1999)].

[11] O. Cata, M. Golterman, S. Peris, JHEP 0508, 076 (2005).

[12] D. Boito, I. Caprini, M. Golterman, K. Maltman, S. Peris, Phys. Rev. D 97, 054007 (2018).

[13] Yu. A. Simonov, Phys. Atom. Nucl. 65, 135 (2002).

[14] S. S. Afonin, Phys. Lett. B 576, 122 (2003).

[15] S. S. Afonin, Nucl. Phys. B 779, 13 (2007).

[16] S. S. Afonin, A. A. Andrianov, V. A. Andrianov, D. Espriu, JHEP 0404, 039 (2004).

[17] S. S. Afonin, A. A. Andrianov, V. A. Andrianov, D. Espriu, Int. J. Mod. Phys. A 21, 885 (2006).

[18] S. S. Afonin, D. Espriu, JHEP 0609, 047 (2006).

[19] S. Beane, Phys. Rev. D 64, 116010 (2001).

[20] M. Golterman, S. Peris, JHEP 0101, 028 (2001).

[21] M. Golterman, S. Peris, B. Phily, E. De Rafael, JHEP 0201, 024 (2002).

[22] S. S. Afonin, PMC Phys. A 3, 1 (2009).

[23] V. I. Shevchenko, Yu. A. Simonov, Phys. Rev. D 70, 074012 (2004).

[24] M. Golterman, S. Peris, Phys. Rev. D 67, 096001 (2003).

[25] P. Masjuan, S. Peris, JHEP 0705, 040 (2007).

[26] E. R. Arriola, W. Broniowski, Phys. Rev. D 73, 097502 (2006).

[27] E. R. Arriola, W. Broniowski, Eur. Phys. J. A 31, 739 (2007).

[28] E. R. Arriola, W. Broniowski, Phys. Rev. D 81, 054009 (2010).

[29] J. J. Sanz-Cillero, Nucl. Phys. B 732, 136 (2006). 
[30] J. Mondejar, A. Pineda, JHEP 0710, 061 (2007).

[31] A. A. Andrianov, D. Espriu, Phys. Lett. B 671, 275 (2009).

[32] S. S. Afonin, T. D. Solomko, Eur. Phys. J. C 76, 678 (2016).

[33] S. S. Afonin, T. D. Solomko, Int. J. Mod. Phys. A 33, 1850069 (2018).

[34] S. S. Afonin, T. D. Solomko, Int. J. Mod. Phys. A 33, 1850115 (2018).

[35] S. S. Afonin, T. D. Solomko, Acta Phys. Polon. Supp. 11, 469 (2018).

[36] S. S. Afonin, T. D. Solomko, EPJ Web of Conferences 191, 04004 (2018).

[37] D. LaCourse, M. G. Olsson, Phys. Rev. D 39, 2751 (1989).

[38] A. Yu. Dubin, A. B. Kaidalov, Yu. A. Simonov, Phys. Lett. B 323, 41 (1994).

[39] G. V. Efimov, S. N. Nedelko, Phys. Rev. D 51, 176 (1995).

[40] R. Ricken, M. Koll, D. Merten, B. C. Metsch, H. R. Petry, Eur. Phys. J. A 9, $221(2000)$.

[41] Yu. S. Kalashnikova, A. V. Nefediev, Yu. A. Simonov, Phys. Rev. D 64, 014037 (2001).

[42] T. J. Allen, C. Goebel, M. G. Olsson, S. Veseli, Phys. Rev. D 64, 094011 (2001).

[43] M. Baker, R. Steinke, Phys. Rev. D 65, 094042 (2002).

[44] A. M. Badalian, B. L. G. Bakker, Y. A. Simonov, Phys. Rev. D 66, 034026 (2002).

[45] F. Buisseret, Phys. Rev. C 76, 025206 (2007).

[46] D. Ebert, R. N. Faustov, V. O. Galkin, Phys. Rev. D 79, 114029 (2009).

[47] J. Sonnenschein, D. Weissman, JHEP 1408, 013 (2014).

[48] S. S. Afonin, Teor. Mat. Fiz. 184, 418 (2015).

[49] S. S. Afonin, arXiv:1705.01899 [hep-ph].

[50] S. S. Afonin, arXiv:1808.07363 [hep-ph].

[51] J. Sonnenschein, Prog. Part. Nucl. Phys. 92, 1 (2017). 
[52] D. Jia, C. Q. Pang, A. Hosaka, Int. J. Mod. Phys. A 32, 1750153 (2017).

[53] A. V. Anisovich, V. V. Anisovich, A. V. Sarantsev, Phys. Rev. D 62, 051502(R) (2000).,

[54] D. V. Bugg, Phys. Rep. 397, 257 (2004).

[55] E. Klempt, A. Zaitsev, Phys. Rep. 454, 1 (2007).

[56] M. Shifman, A. Vainshtein, Phys. Rev. D 77, 034002 (2008).

[57] S. S. Afonin, Phys. Lett. B 639, 258 (2006).

[58] S. S. Afonin, Mod. Phys. Lett. A 22, 1359 (2007).

[59] S. S. Afonin, Int. J. Mod. Phys. A 22, 4537 (2007).

[60] S. S. Afonin, Phys. Rev. C 76, 015202 (2007).

[61] S. S. Afonin, Int. J. Mod. Phys. A 23, 4205 (2008).

[62] S. S. Afonin,Mod. Phys. Lett. A 23, 3159 (2008).

[63] D. M. Li, B. Ma, Y. X. Li, Q. K. Yao, H. Yu, Eur. Phys. J. C 37, 323 (2004).

[64] S. S. Afonin, Eur. Phys. J. A 29, 327 (2006).

[65] P. Masjuan, E. Ruiz Arriola, W. Broniowski, Phys. Rev. D 85, 094006 (2012).

[66] A. Karch, E. Katz, D. T. Son, M. A. Stephanov, Phys. Rev. D 74, 015005 (2006).

[67] S. J. Brodsky, G. F. de Teramond, H. G. Dosch, J. Erlich, Phys. Rept. 584, 1 (2015).

[68] S. S. Afonin, Phys. Lett. B 719, 399 (2013).

[69] S. S. Afonin, Phys. Lett. B 678, 477 (2009).

[70] S. S. Afonin, Mod. Phys. Lett. A 32, 1750155 (2017).

[71] S. S. Afonin, Acta Phys. Polon. Supp. 9, 597 (2016).

[72] S. S. Afonin, Int. J. Mod. Phys. A 25, 5683 (2010).

[73] S. S. Afonin, Adv. High Energy Phys. 2017, 8358473 (2017). 
[74] J. J. Sakurai, Ann. Phys. 11, 1 (1960).

[75] A. Bramon, M. Greco, Lett. Nuovo Cimento 3, 693 (1972).

[76] A. Bramon, E. Etim, M. Greco, Phys. Lett. B 41, 609 (1972).

[77] J. J. Sakurai, Phys. Lett. B 46, 207 (1973).

[78] V. K. Henner, T. S. Belozerova, Phys. Atom. Nucl. 59, 1851 (1996). 61, 119 (1998).

[79] V. A. Andrianov, S. S. Afonin, Phys. Atom. Nucl. 65, 1862 (2002).

[80] S. N. Nedelko, V. E. Voronin, Phys. Rev. D 93, 094010 (2016).

[81] A. M. Badalian, B. L. G. Bakker, Phys. Rev. D 93, 074034 (2016).

[82] J. F. Jiang, S. L. Zhu, Phys. Rev. D 92, 074002 (2015).

[83] S. S. Afonin, I. V. Pusenkov, Phys. Rev. D 90, 094020 (2014).

[84] S. S. Afonin, I. V. Pusenkov, Mod. Phys. Lett. A 29, 1450193 (2014).

[85] S. S. Afonin, I. V. Pusenkov, EPJ Web Conf. 125, 04006 (2016).

[86] P. Masjuan, E. Ruiz Arriola, W. Broniowski, EPJ Web Conf. 73, 04021 (2014).

[87] M. A. Shifman, A. I. Vainstein, V. I. Zakharov, Nucl. Phys. B 147, 385, 448 (1979).

[88] J. R. Pelaez, Phys. Rev. Lett. 92, 102001 (2004).

[89] Z. H. Guo, J. A. Oller, Phys. Rev. D 84, 034005 (2011).

[90] J. Nieves, A. Pich, E. Ruiz Arriola, Phys. Rev. D 84, 096002 (2011).

[91] E. Ruiz Arriola, W. Broniowski, Phys. Rev. D 78, 034031 (2008).

[92] P. Masjuan, E. Ruiz Arriola, W. Broniowski, Phys. Rev. D 87, 014005 (2013).

[93] B. Bisschoff, C. A. Dominguez, L. A. Hernandez, Mod. Phys. Lett. A 32, 1750036 (2017). 